\title{
Acute Abdomen-A Clinical Presentation of MIS-C in Children
}

\author{
Emrah Gün ${ }^{1}$ Tanıl Kendirli ${ }^{1}$ Edin Botan ${ }^{1}$ Berrin Demir ${ }^{2}$ () \\ Ömer Suat Fitoz ${ }^{2} \odot$ Ergin Ciftci ${ }^{4}$ Ercan Tutar ${ }^{5}$ \\ ${ }^{1}$ Department of Pediatric Intensive Care, Division of Pediatric \\ Intensive Care, Ankara University School of Medicine, Ankara, Turkey \\ ${ }^{2}$ Department of Pediatric Radiology, Ankara University School of \\ Medicine, Ankara, Turkey \\ ${ }^{3}$ Department of Pediatric Surgery, Ankara University School of \\ Medicine, Ankara, Turkey \\ ${ }^{4}$ Department of Pediatric Infectious Diseases, Ankara University \\ School of Medicine, Ankara, Turkey \\ ${ }^{5}$ Department of Pediatric Cardiology, Cebeci Hospitals, Ankara \\ University School of Medicine, Ankara, Turkey \\ J Pediatr Infect Dis 2022;17:24-32.
}

Ergun Ergün ${ }^{3} \quad$ Halil Özdemir ${ }^{4}$

Address for correspondence Emrah Gün, MD, Department of Pediatrics, Division of Pediatric Intensive Care, Ankara University Faculty of Medicine, Ankara, Turkey (e-mail: emrhgn@hotmail.com).

\author{
Abstract \\ Keywords \\ - multisystem \\ inflammatory \\ syndrome in children \\ - COVID-19 \\ - intestinal \\ inflammation \\ - appendicitis \\ - children
}

Objective Multisystemic inflammatory syndrome in children (MIS-C) is characterized by persistent fever, systemic hyperinflammation, and multiple-organ dysfunction. There are a few reports about MIS-C presenting with acute abdomen. The aim of this study was to demonstrate the clinical characteristics and treatment options for MIS-C-related acute abdomen and appendicitis.

Methods This was a retrospective study conducted between April 2020 and October 2020 in our pediatric intensive care unit in Turkey. Patients between the ages of 1 month and 18 years who presented with acute abdomen and were ultimately diagnosed with MIS-C were included.

Results Seven patients with a median age of 12.5 (interquartile range 10.5-13) years were enrolled. Four were females. The most frequent symptoms were fever, abdominal pain, and vomiting. Three patients had involvement of the appendix that required surgical intervention. All pathology reports were compatible with appendicitis. The other patients also had an acute abdomen. One patient had malignant hyperthermia during induction of anesthesia, so surgery was postponed and medical management was commenced. The clinical picture regressed with immunomodulation. All patients were treated with intravenous immunoglobulin and steroids. Four patients with acute abdomens improved with immunomodulation, and surgery was not needed.

Conclusion MIS-C may present with an acute abdomen. Immunomodulation should be considered instead of surgery if the clinical course is not complicated.

\section{Introduction}

Initial reports of pediatric coronavirus disease 2019(COVID-19) showed that the disease was usually milder in children than it was in adults. ${ }^{1}$ However, some reports were issued in April 2020 regarding pediatric patients presenting with a severe Kawasakilike syndrome associated with recently proven or suspected severe acute respiratory syndrome coronavirus 2 (SARS-CoV-2) received

May 12, 2021

accepted after revision

September 30, 2021

published online

December 6, 2021 (c) 2021. Thieme. All rights reserved.

Georg Thieme Verlag KG,

Rüdigerstraße 14,

70469 Stuttgart, Germany
DOI https://doi.org/

10.1055/s-0041-1739394.

ISSN 1305-7707. 
infection. ${ }^{1}$ This clinical condition was named pediatric inflammatory multisystem syndrome temporally associated with SARS-CoV-2 (PIMS-TS) by the Royal College of Pediatrics and Child Health. Subsequently, this clinical entity was named multisystem inflammatory syndrome in children (MIS-C) by the World Health Organization and the Centers for Disease Control and Prevention (CDC) after similar cases were reported throughout the world in May 2020. ${ }^{2}$ These patients presented with recurrent fever and gastrointestinal (GI) symptoms including abdominal pain, vomiting, diarrhea; mucocutaneous symptoms including rash, conjunctivitis; and neurological symptoms such as headache, irritability, and encephalopathy. ${ }^{3,4}$

MIS-C is characterized by persistent fever, systemic hyperinflammation, and multiple-organ dysfunction. ${ }^{5}$ These patients may rapidly progress to hypotension and shock with cardiac and other end-organ injury. ${ }^{3}$ Occasional reports of MIS-C presenting with an acute abdomen may be found. ${ }^{5}$ We hereby presented seven patients who were admitted to the pediatric intensive care unit (PICU) with an acute abdomen and were subsequently diagnosed with MIS-C.

\section{Material and Methods}

This study was a retrospective cohort study between April 2020 and October 2020 in our PICU in Ankara, Turkey. Patients between the ages of 1 month and 18 years who presented with an acute abdomen and were ultimately diagnosed with MIS-C were included. Ethical approval was obtained from the local Institutional Review Board.

All included patients met the CDC MIS-C diagnostic criteria (-Table 1). Clinical and radiologic data were obtained from patients' charts and electronic records. ${ }^{6}$ MIS-C is usually treated with steroids and intravenous immunoglobulin (IVIG) as immunomodulator therapy, enoxaparin and acetylsalicylic acid as anticoagulants, and, occasionally, anti-cytokine agents such as tocilizumab and infliximab are employed. ${ }^{7}$

A thickened, non-compressible appendix larger than $6 \mathrm{~mm}$ in diameter was considered as acute appendicitis on abdominal ultrasound. ${ }^{8}$ On abdominal computed tomography (CT), an enlarged appendix greater than $6 \mathrm{~mm}$ with a thickened wall $(>2 \mathrm{~mm})$, peri-appendiceal inflammation, appendicolith, appendiceal abscess, or abdominal free fluid were interpreted as acute appendicitis. ${ }^{8}$

Age, gender, hospital length of stay (LOS), underlying medical conditions, abdominal pain, diarrhea, thrombocytopenia, lymphopenia, myocarditis, congestive heart failure, shock, hypotension, pericardial effusion, coronary artery dilatation or aneurysm, arrhythmia, rash, mucocutaneous lesion, conjunctival involvement, cough, dyspnea, chest pain, pneumonia, acute respiratory distress syndrome, pleural effusion, infiltration on chest X-ray, headache, seizure, presence of acute renal injury, invasive or noninvasive mechanical ventilation, complete blood count, biochemical parameters and acute phase reactants, coagulation parameters, cardiac markers, treatments (IVIG, steroid, tocilizumab, anakinra, anticoagulant therapy, antiplatelet therapy, vasopressors), CT results, magnetic resonance imaging results, and echocardiography results were recorded retrospectively from patient charts and electronic hospital records.

The vasoactive inotropic score was calculated using the following formula: (dopamine dose $[\mu \mathrm{g} / \mathrm{kg} / \mathrm{min}]+$ dobutamine dose $[\mu \mathrm{g} / \mathrm{kg} / \mathrm{min}]+100 \times$ epinephrine dose $[\mu \mathrm{g} / \mathrm{kg} /$ min $]+10 \times$ milrinone dose $[\mu \mathrm{g} / \mathrm{kg} / \mathrm{min}]+10,000 \times$ vasopressin dose [unit $/ \mathrm{kg} / \mathrm{min}]+100 \times$ norepinephrine dose $[\mu \mathrm{g} / \mathrm{kg} / \mathrm{min}]) .{ }^{9}$ Obesity was defined as a body mass index $\geq 95$ th percentile for age in children.

The data were analyzed using SPSS 23.0 (Statistical Package for Social Sciences for Windows, Inc., United States). The expression $n$ (\%) was used for categorical variables, and median values and interquartile ranges (IQR 25-75) were used for continuous variables.

\section{Results}

Nineteen patients were admitted to the PICU due to MIS-C. Seven patients had an acute abdomen and were diagnosed with MIS-C on follow-up. The median age of these seven patients was 12.5 (IQR 10.5-13) years. Four patients were girls. Two patients had obesity. SARS-CoV-2 serology was positive in two patients, and three patients had a history of COVID-19 polymerase chain reaction (PCR) positivity. Serology was negative in the other two patients, but they had a history of exposure to COVID-19 in the family 1 month prior.

Seven patients had abdominal pain and vomiting, five patients had rash, two patients had conjunctivitis, five patients had headaches, six patients needed inotropic support, seven patients had persistent tachycardia, and one patient had arrhythmia (-Table 2). All seven patients had leukocytosis, lymphopenia, elevated CRP and procalcitonin levels, elevated troponin-T and pro-BNP levels, and elevated ferritin and D-dimer levels. Two patients had thrombocytopenia. Two had high creatinine $(>1 \mathrm{mg} / \mathrm{dL})$, and six had elevated lactate dehydrogenase. None had hypoalbuminemia.

All patients received oxygen by mask, and two needed biphasic positive airway pressure support. No patients were intubated. All patients survived to PICU discharge. All patients received corticosteroids, five patients received IVIG, two patients received pulse steroids, two patients received acetylsalicylic acid, and two patients received enoxaparin.

Three patients underwent appendectomy. These patients had postoperative pulmonary edema. Case 1 and case 6 had pulmonary congestion on chest X-ray (-Figs. 1A and 2). Case 2 and case 6 had acute kidney injury, which improved after immunomodulation. Their pathology reports showed lymphoid hyperplasia and severe inflammation and were consistent with acute appendicitis.

Case 1 was a 12-year-old male patient who had undergone appendectomy and was admitted to the PICU after he had rash, bloody vomiting, hypotension, and circulatory failure in the operating room. The patient's SARS-CoV-2 serology was negative, but the patient had a history of contact with COVID-19 4 weeks ago. The appendix of case 1 appeared 


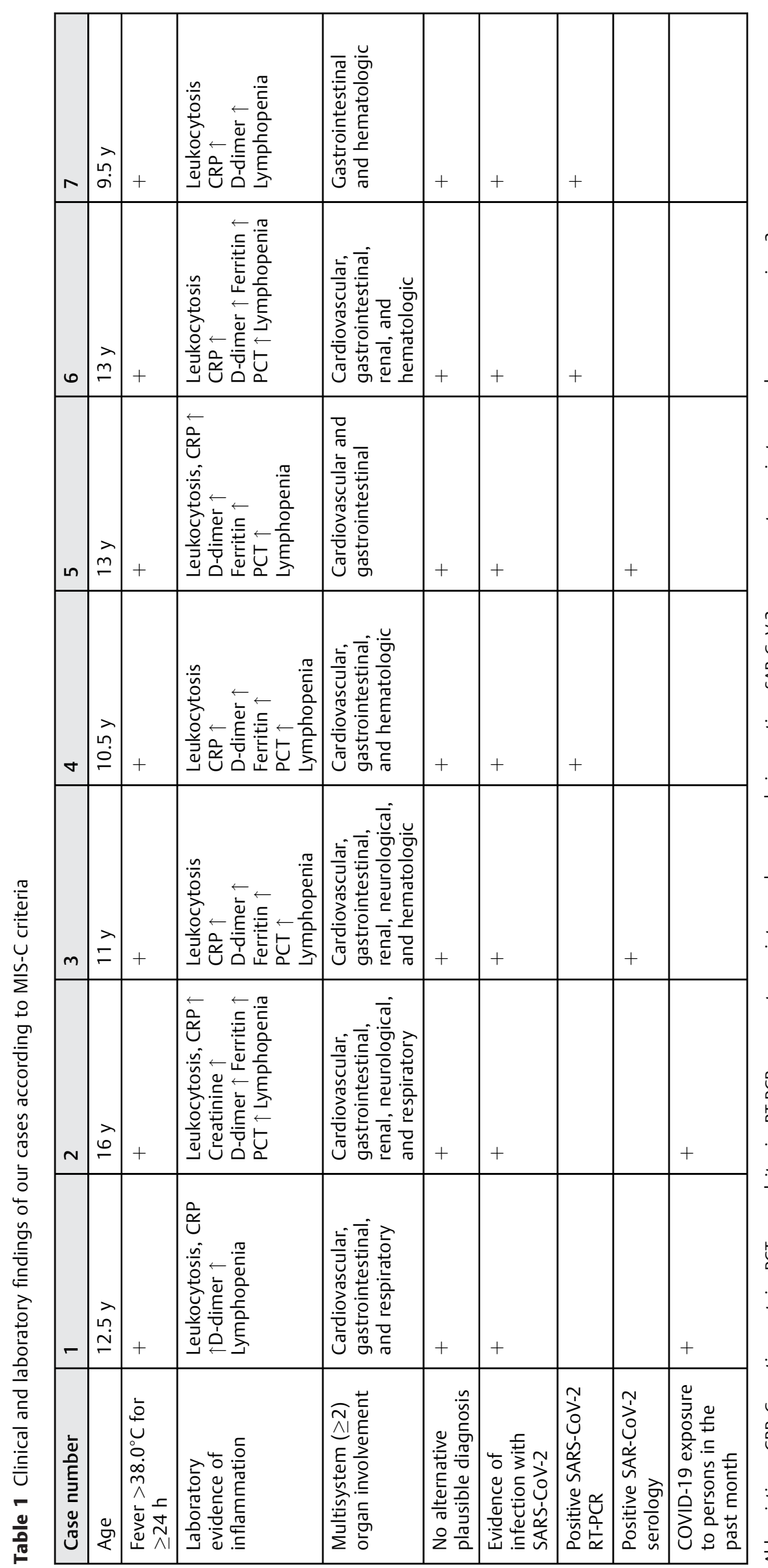


A Clinical Presentation of MIS-C in Children Gün et al. 27

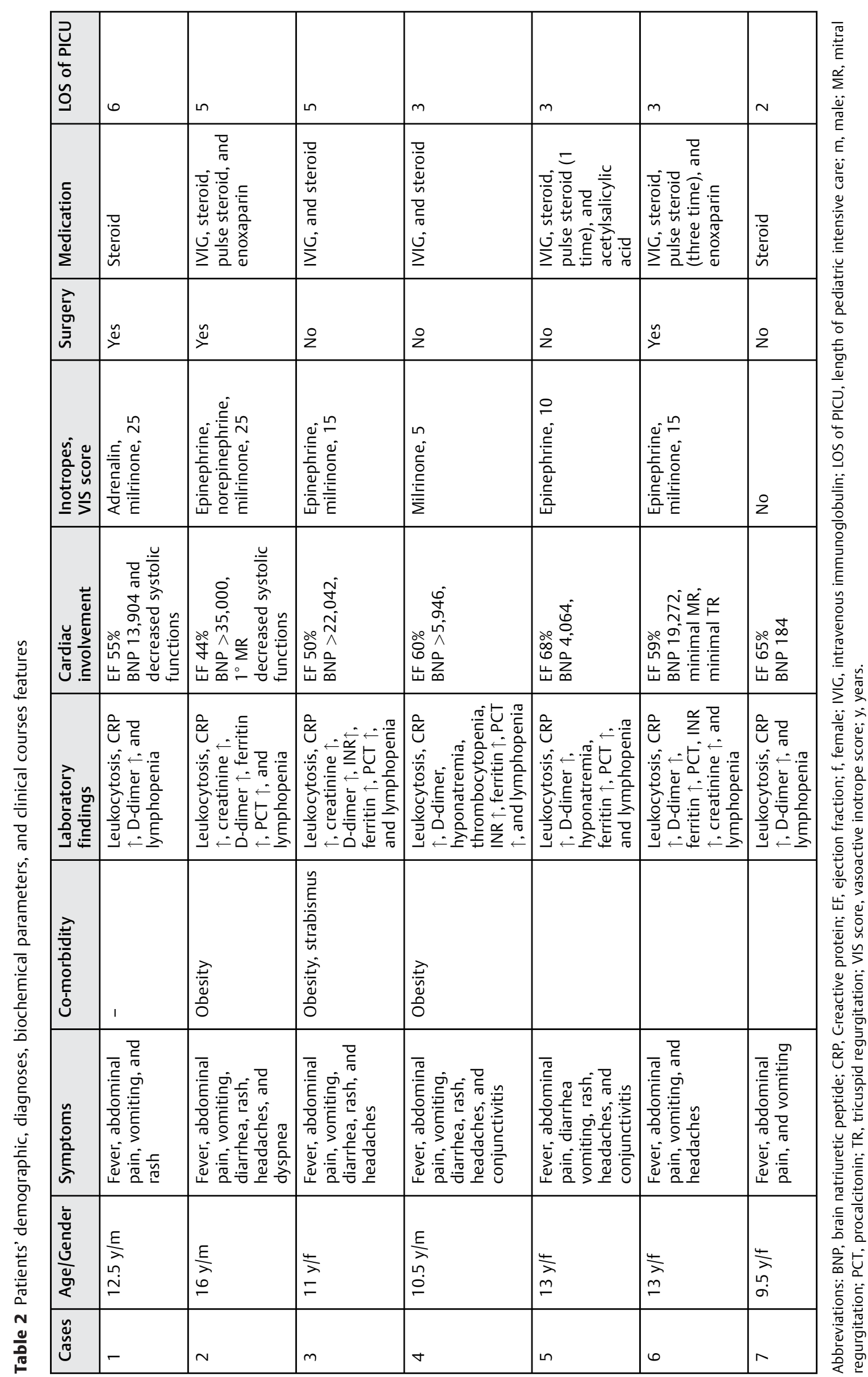




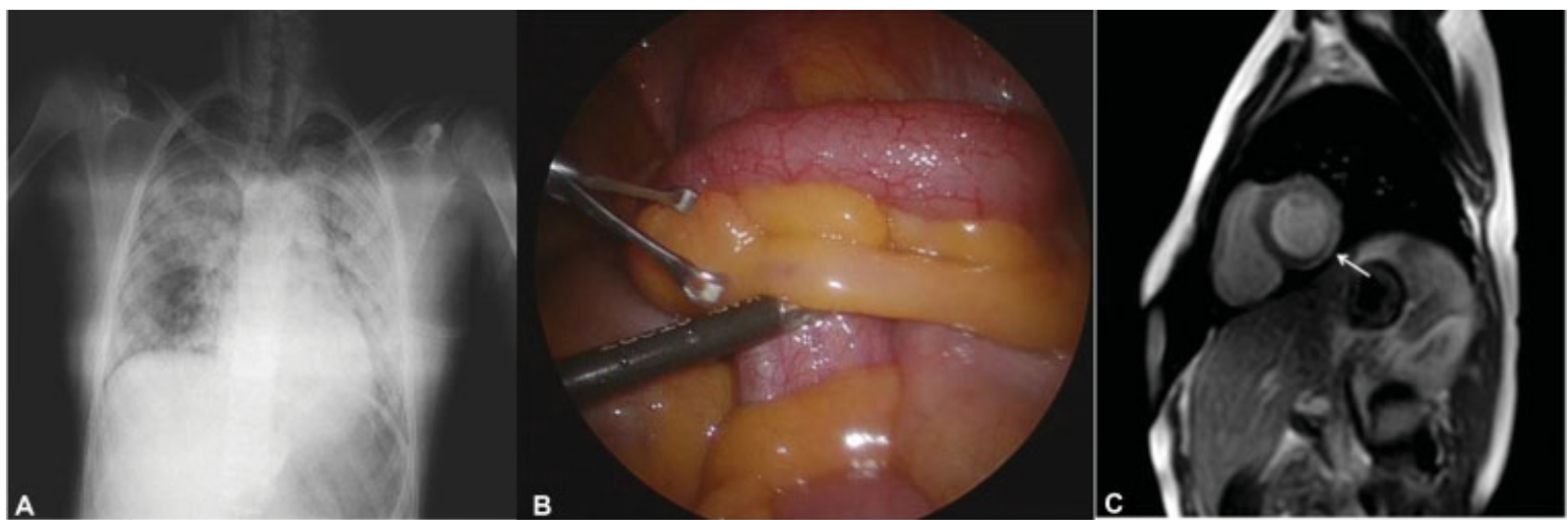

Fig. 1 (A) In patient 1, chest X-ray graph demonstrates diffuse infiltration in all lung areas due to heart failure. (B) In patient 1, there was inflammation and hyperemia in the appendix. (C) In case 1, short-axis late gadolinium-enhanced cardiac MRI; contrast enhancement consistent with inflammation in myocardium is seen at the lateral basal part of left ventricle (white arrow).

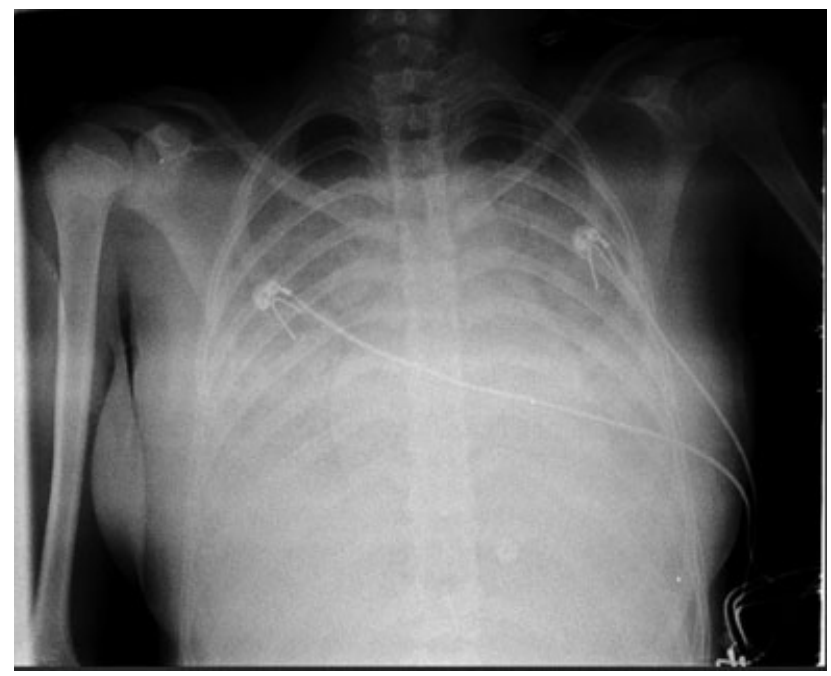

Fig. 2 In patient 6, chest X-ray graph demonstrates congestion findings.

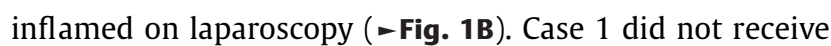
MIS-C treatment. MIS-C was diagnosed due to the presence of myocarditis on cardiac MRI after discharge and its clinical similarity to other patients (-Fig. 1C).

Case 2 was a 16-year-old male with no history of chronic illness other than obesity who had been admitted to the referring hospital with complaints of fever, abdominal pain, confusion, weakness, diarrhea, and a rash. His examination findings were consistent with an acute abdomen, and his abdominal CT scan revealed an increased $(1.1 \mathrm{~cm})$ diameter of the appendix and mesenteric contamination. He underwent appendectomy and was admitted to the PICU and needed vasoactive infusions due to shock. ECG revealed ST depression in leads V3 to V6, and echocardiogram revealed an ejection fraction of $40 \%$. He was started on IVIG $2 \mathrm{~g} / \mathrm{kg}$, methylprednisolone, and enoxaparin.

Case 3 was an 11-year-old girl. She was presented with complaints of shock, neurological changes, and skin lesions. Her abdominal ultrasonography (USG) revealed significant inflammation in the right lower quadrant, where a hypodense $2 \times 1.5 \mathrm{~cm}$ tubulonodular area, thought to represent fluid collection or a swollen lymph node, was observed.
There were a few more enlarged lymph nodes nearby. An increased $(1.1 \mathrm{~cm})$ diameter of appendix was noted, but its tip could not be visualized. This was considered to be possibly due to acute appendicitis. Abdominal CT revealed diffuse inflammatory changes in the right lower quadrant, multiple enlarged lymph nodes, a fluid collection that could not be distinguished with certainty from the cecum, and free fluid in the right lower quadrant and pelvis. These findings suggested possible perforated appendicitis (-Fig. 3). When

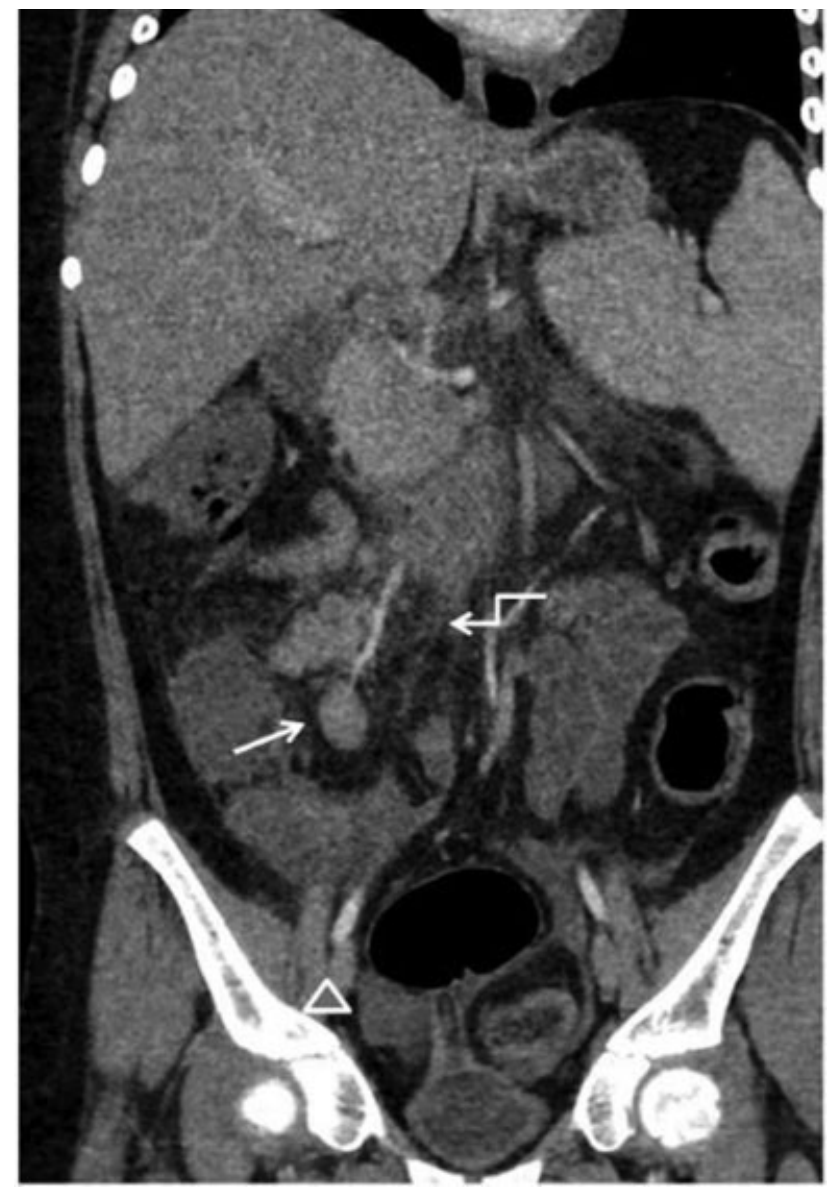

Fig. 3 In patient 3, coronal plane CT of the abdomen; mesentery inflammation (curved arrow), lymphadenomegaly (white arrow), and fluid (arrow head) in pelvic area. 


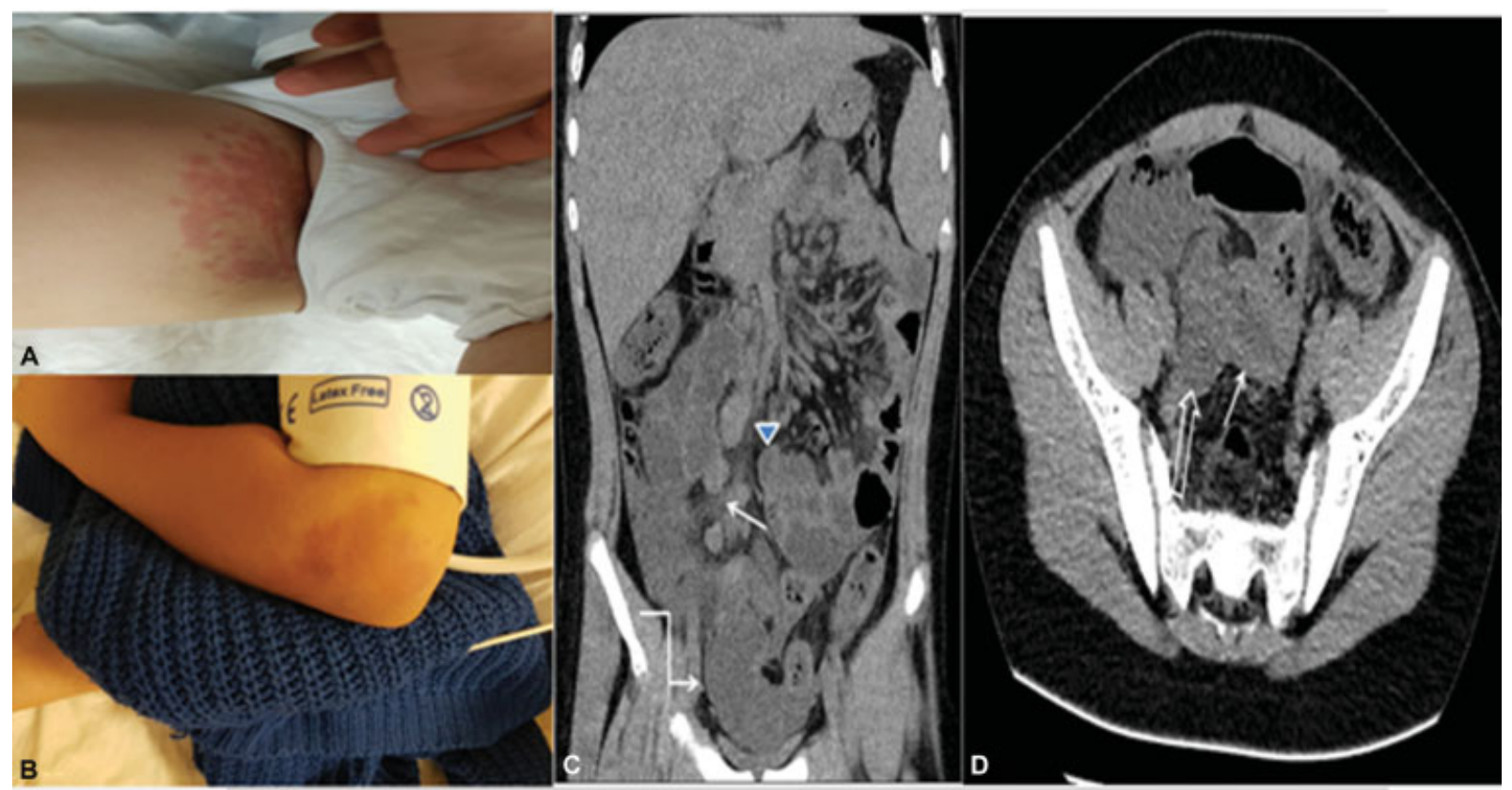

Fig. 4 (A and B) In patient 4, a blanching maculopapular rash on the extremities, right inguinal area, left elbow. (C) In patient 4, non-enhanced coronal reformatted CT image of abdomen; increased mesenteric fat density consistent with mesenteric edema and inflammation (white arrow), mesenteric lymphadenopathy (arrow head), and pelvic fluid (curved arrow). (D) In patient 4, axial CT of image of pelvis showing diffuse thickening of terminal ileum wall (white arrow) and fluid (open arrow).

case 3's SARS-CoV-2 antibody serology was positive, IVIG and methylprednisolone treatment were started. A decrease in acute phase reactants was observed after treatment. Abdominal distension also regressed within 3 days. The patient was transferred to the pediatric surgery ward on the 5th day. Patient 3 was pending a diagnostic laparotomy, but it was ultimately not needed because the clinical findings regressed.

Case 4 was a 10 -year-old male child patient with a history of COVID-19 with a positive SARS-CoV2 PCR test 1 month prior who presented to the pediatric emergency room with fever, rash, and diarrhea for 4 days. His abdomen was tender, and his bowel sounds were hyperactive. Case 4 had skin lesions (-Fig. 4A, 4B). There was terminal ileum involvement, but the appendix could not be visualized on abdominal CT ( - Fig. 4C, 4D). He was started on $2 \mathrm{~g} / \mathrm{kg}$ of IVIG over 2 days and $2 \mathrm{mg} / \mathrm{kg} / \mathrm{d}$ of methylprednisolone. Pleural effusion was noted on lung ultrasound, so furosemide infusion was started. The ejection fraction on echocardiography was 59 to $60 \%$, so milrinone infusion was started.

Case 5, a previously healthy 14 -year-old girl, was admitted to the emergency room with fever, weakness, headache, abdominal pain, diarrhea, and rash. She had been febrile for 3 days and had increasingly intense abdominal pain for 2 days. She had a maculopapular rash on her abdomen, back, and buttocks. She had abdominal distention and guarding. Enlarged right parailiac lymph nodes with heterogeneous echogenicity were noted on ultrasound. Her SARS-CoV-2 serology was positive. She was diagnosed with MIS-C, and she received IVIG ( $1 \mathrm{~g} / \mathrm{kg} / \mathrm{d}$ for 2 days), acetylsalicylic acid, one dose of pulse steroids $(1 \mathrm{~g})$, and methylprednisolone $2 \mathrm{mg} / \mathrm{kg} / \mathrm{d}$. Her abdominal distention regressed on the second day of treatment, and she tolerated enteral feeds. She was transferred to the pediatric ward on day 3 of hospitalization.

Case 6 was a 13-year-old girl child who was admitted with intermittent abdominal pain, fever, and diarrhea for the preceding 5 days. The pediatric surgery team was consulted due to the findings of mesenteric lymphadenitis and acute appendicitis on abdominal USG. She had a positive COVID-19 PCR result 1 month prior, and her SARS-CoV2 serology was positive. She did not undergo immediate surgery and was started on IVIG and methylprednisolone. However, her fever persisted and her abdomen became distended on follow-up, so she underwent surgery with a preliminary diagnosis of perforated appendicitis. Her pathology report was consistent with appendicitis and localized peritonitis.

Case 7 was a 9.5-year-old girl with a history of COVID-19 2 months prior who presented with fever, abdominal pain, and vomiting for 4 days. Her examination findings were consistent with an acute abdomen, and her abdominal USG revealed an increased $(12 \mathrm{~mm})$ appendiceal diameter. She had malignant hyperthermia upon induction of anesthesia, so her surgery was postponed. She received dantrolene and immunomodulation with IVIG, and methylprednisolone was commenced. Improvement was noted with medical management, and surgery was not needed.

The median PICU LOS was 3 (IQR 3-5) days. All patients survived and had good cardiac function on PICU discharge.

\section{Discussion}

We hereby report our experience with seven patients who had an acute abdomen caused by MIS-C, which is an entity that is distinct from acute COVID-19 infection. Three patients underwent appendectomy, and their pathology results were 


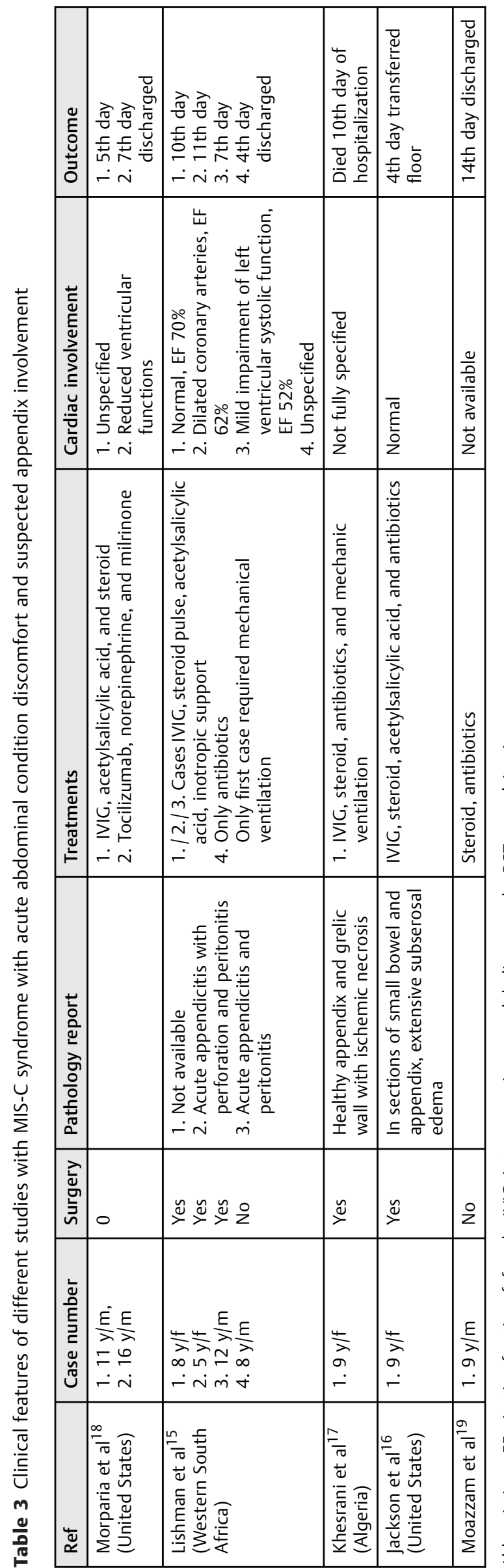

consistent with appendicitis. Four patients were managed with immunomodulation.

Initial reports of MIS-C were similar to Kawasaki disease (KD) in that the primary target organ was the heart, but subsequently, reports of this syndrome mimicking acute appendicitis and acute abdomen emerged. GI involvement can also occur with KD, but the underlying mechanisms are not clear. In the study by Fabi et al ${ }^{10} 106$ of 302 patients with KD had GI involvement and children younger than 6 months were more likely to have GI involvement. However, this study did not report how many patients required GI surgery. MIS-C seems to occur at a later age than $\mathrm{KD}$, and $\mathrm{GI}$ involvement is much more common in MIS-C. In the study by García-Salido et al, ${ }^{11} 60 \%$ of patients with MIS-C had GI involvement.

Acute abdomens may be caused by an infection, inflammation, vascular occlusion, or obstruction. An acute abdomen presents with sudden onset of abdominal pain, usually accompanied by nausea or vomiting, and it requires urgent attention and treatment. ${ }^{12}$ MIS-C, a post-infectious condition, can also present with an acute abdomen, caused by inflammation. The most common GI symptoms in MIS-C patients were reported to be abdominal pain, nausea, and vomiting. ${ }^{9,13}$ While any segment of the GI tract may be involved, inflammation in the ileum and colon is the most common ( - Table 3 ). Progressive intestinal mural thickening can cause narrowing and obstruction of the intestinal lumen. Intestinal inflammation usually resolves with medical treatment but, in rare cases, surgery may be needed. ${ }^{14}$ Moreover, it has been stated that acute appendicitis may be associated with appendicular arterial vasculitis in MIS-C. ${ }^{15}$ In the report by Lishman et al, ${ }^{1}$ three patients had undergone appendectomy with SARS-CoV-2 serology positivity. Two of three patients had a perforated appendix, and one patient had hyperemia in the appendix. Similarly, in our report, patients 1 and 2 had appendiceal involvement that required surgical intervention. Pathology reports of all three cases were compatible with appendicitis.

In the study of Jackson et al, ${ }^{16}$ a 9 -year-old girl had acute abdominal pain. She was diagnosed with appendicitis by abdominal ultrasonography and, consequently, underwent appendectomy. This patient was reported to have positive SARS-CoV-2 serology and started postoperatively on IVIG and steroids for MIS-C. The pathology report was consistent with extensive subserosal edema of the small bowel and appendix and necrotizing lymphadenitis of mesenteric lymph nodes. ${ }^{16}$

However, there may also be a pseudoappendicular syndrome as in the 9-year-old MIS-C case reported by Khesrani et al. ${ }^{17}$ It was reported that the patient also had shock, neurological abnormalities, and skin lesions. ${ }^{17}$ They stated that the patient had ischemic lesions in the intestines and a healthy appendix on laparotomy. ${ }^{17}$ In our report, patients 1 , 2 , and 6 who underwent appendectomy had shock requiring vasoactive infusions. Case 7 could not undergo appendectomy due to malignant hyperthermia during induction and ultimately improved on immunomodulation. She had appendicitis on ultrasound, but she did not need surgery. She had a proven COVID-19 PCR positivity 2 months prior. 
Similarly, cases 3, 4, and 5 also improved on immunomodulation, precluding the need for surgery.

In the report by Sahn et $\mathrm{al}^{14}$ one patient had severe stenosis of the terminal ileum with circumferentially increased intestinal wall thickness. The resulting obstructive symptoms resolved with medical treatment. ${ }^{14}$ In this study, abdominal imaging findings of 19 MIS-C patients were also reported. Seven patients had inflammatory bowel changes, and four patients had prominent terminal ileitis and cecum involvement. ${ }^{14}$

In the study of Morporia et al, ${ }^{18}$ one patient had appendiceal dilation and enlarged lymph nodes in the right hemiabdomen. Another patient had a normal appendix, mural thickening, and enhancement of terminal ileum, diffuse mesenteric lymphadenopathy. Both patients recovered with immunomodulation. ${ }^{17}$

In another study, $34.1 \%$ of 44 MIS-C patients underwent abdominal imaging. ${ }^{5}$ In this study, two patients had mesenteric lymphadenitis, six patients had acalculous cholecystitis, and six patients had ascites. It was reported that three patients had intestinal wall thickening similar to inflammatory bowel disease. One of these three patients had intense right lower quadrant pain, fever, and rash. On MR imaging, severe concentric mural thickening, edema and extensive mesenteric fat edema, hyperenhancement of a short segment of the terminal ileum, and, similarly, mural thickening of the wall in the rectosigmoid colon were reported. It was reported that the other two patients had nonspecific thickened bowel folds in the right lower quadrant on ultrasound. ${ }^{5}$

In conclusion, MIS-C, an increasingly frequent disease, should be considered in patients presenting with an acute abdomen throughout the pandemic. Abdominal involvement in MIS-C is more common than in KD, but it may be challenging to distinguish one from the other. Serology for SARS-CoV-2 may be helpful in discriminating abdominal involvement of MIS-C and acute appendicitis. These patients may improve with medical treatment and thus surgery may not be required, as seen in some of our patients.

\section{What Is Known}

- MIS-C is characterized by persistent fever, systemic hyperinflammation, and multiple-organ dysfunction.

- MIS-C may rapidly progress to hypotension and shock with cardiac and other end-organ injury.

\section{What Is New}

- MIS-C may present as an acute abdominal condition.

- Initially, immunomodulatory therapy should be considered instead of surgery in the first-step approach if the cases are not complicated.

Consent to Participate

Approval was obtained from the family of the participants.
Ethical Approval

Ethical committee approval was received for this study from the Ethics Committee of Ankara University Medical Faculty by the number i2-143-21.

\section{Authors' Contributions}

T.K. and E.G. were responsible for the concept; E.G., E.D., E.E., and B.A. for the design; T.K., E.Ç., E.T., H.Ö., and S.F. for supervision; E.G., T.K., and E.D. for materials; E.G., T.K., and E.D. for data collection and/or processing; T.K. and E.G. for the analysis and/or interpretation; T.K., E.G., and E.D. for the literature review; T.K. and E.G. for writing; and T.K., E.T., E.Ç., E.E., S.F., and H.Ö. for critical review.

Funding

None.

Conflict of Interest

None declared.

\section{References}

1 Diorio C, Henrickson SE, Vella LA, et al. Multisystem inflammatory syndrome in children and COVID-19 are distinct presentations of SARS-CoV-2. J Clin Invest 2020;130(11):5967-5975

2 Lee PY, Day-Lewis M, Henderson LA, et al. Distinct clinical and immunological features of SARS-CoV-2-induced multisystem inflammatory syndrome in children. J Clin Invest 2020;130(11): 5942-5950

3 Blumfield E, Levin TL, Kurian J, Lee EY, Liszewski MC. Imaging findings in multisystem inflammatory syndrome in children (MIS-C) associated with COVID-19. Am J Roentgenol 2021;216 (02):507-517

4 Nakra NA, Blumberg DA, Herrera-Guerra A, Lakshminrusimha S. Multi-system inflammatory syndrome in children (MIS-C) following SARS-CoV-2 infection: review of clinical presentation, hypothetical pathogenesis, and proposed management. Children (Basel) 2020;7(07):E69

5 Miller J, Cantor A, Zachariah P, Ahn D, Martinez M, Margolis KG. Gastrointestinal symptoms as a major presentation component of a novel multisystem inflammatory syndrome in children that is related to coronavirus disease 2019: a single center experience of 44 cases. Gastroenterology 2020;159(04):1571-1574.e2

6 CDC Health Alert Network. Multisystem inflammatory syndrome in children (MIS-C) associated with coronavirus disease 2019 (COVID-19). Accessed October 22, 2020 at: https://emergency. cdc.gov/han/2020/han00432.asp

7 Feldstein LR, Rose EB, Horwitz SM, et al; Overcoming COVID-19 Investigators CDC COVID-19 Response Team. Multisystem inflammatory syndrome in U.S. children and adolescents. N Engl J Med 2020;383(04):334-346

8 Gadiparthi R, Waseem M. Pediatric Appendicitis. Treasure Island, FL: StatPearls Publishing 2020; LLC

9 Jonat B, Gorelik M, Boneparth A, et al. Multisystem inflammatory syndrome in children associated with coronavirus disease 2019 in a children's hospital in New York City: patient characteristics and an institutional protocol for evaluation, management, and followup. Pediatr Crit Care Med 2021;22(03):e178-e191

10 Fabi M, Corinaldesi E, Pierantoni L, et al. Gastrointestinal presentation of Kawasaki disease: a red flag for severe disease? PLoS One 2018;13(09):e0202658

11 García-Salido A, de Carlos Vicente JC, Belda Hofheinz S, et al; Spanish Pediatric Intensive Care Society working group on SARS$\mathrm{CoV}-2$ infection. Severe manifestations of SARS-CoV-2 in children 
and adolescents: from COVID-19 pneumonia to multisystem inflammatory syndrome: a multicentre study in pediatric intensive care units in Spain. Crit Care 2020;24(01):666

12 Patterson JW, Kashyap S, Dominique E. Acute Abdomen. Treasure Island, FL: StatPearls Publishing; 2021

13 Kaushik S, Aydin SI, Derespina KR, et al. Multisystem inflammatory syndrome in children associated with severe acute respiratory syndrome coronavirus 2 infection (MIS-C): a multi-institutional study from New York City. J Pediatr 2020;224:24-29

14 Sahn B, Eze OP, Edelman MC, et al. Features of intestinal disease associated with COVID-related multisystem inflammatory syndrome in children. J Pediatr Gastroenterol Nutr 2021;72(03):384-387

15 Lishman J, Kohler C, de Vos C, et al. Acute appendicitis in multisystem inflammatory syndrome in children with COVID-19. Pediatr Infect Dis J 2020;39(12):e472-e473
16 Jackson RJ, Chavarria HD, Hacking SM. A case of multisystem inflammatory syndrome in children mimicking acute appendicitis in a COVID-19 pandemic area. Cureus 2020;12(09): e10722

17 Khesrani LS, Chana K, Sadar FZ, Dahdouh A, Ladjadj Y, Bouguermouh D. Intestinal ischemia secondary to COVID-19. J Pediatr Surg Case Rep 2020;61:101604

18 Morparia K, Park MJ, Kalyanaraman M, McQueen D, Bergel M, Phatak T. Abdominal imaging findings in critically ill children with multisystem inflammatory syndrome associated with COVID-19. Pediatr Infect Dis J 2021;40(02):e82-e83

19 Moazzam Z, Salim A, Hilal K, Arshad M. COVID-19 multisystem inflammatory syndrome in children (MIS-C) simulating as acute appendicitis: a case report. J Pediatr Adolesc Surgery 2020;1(01): 56-59 